\title{
Traumatic pulmonary artery injury: a review of the recent literature
}

\author{
Youichi Yanagawa, Kouhei Ishikawa, Hiroki Nagasawa, Ikuto Takeuchi, Kei Jitsuiki, Hiromichi Ohsaka, \\ Kazuhiko Omori
}

Department of Acute Critical Care Medicine, Shizuoka Hospital, Juntendo University, Izunokuni 410-2295, Shizuoka, Japan.

Correspondence to: Dr. Youichi Yanagawa, Department of Acute Critical Care Medicine, Shizuoka Hospital, Juntendo University, 1129 Nagaoka, Izunokuni 410-2295, Shizuoka, Japan. E-mail: yyanaga@juntendo.ac.jp

How to cite this article: Yanagawa Y, Ishikawa K, Nagasawa H, Takeuchi I, Jitsuiki K, Ohsaka H, Omori K. Traumatic pulmonary artery injury: a review of the recent literature. Vesse/ P/us 2018;2:1. http://dx.doi.org/10.20517/2574-1209.2017.37

Received: 12 Dec 2017 First Decision: 8 Jan 2018 Revised: 19 Jan 2018 Accepted: 22 Jan 2018 Published: 26 Jan 2018

Science Editor: Mario F. L. Gaudino Copy Editor: Jun-Yao Li Production Editor: Cai-Hong Wang

\begin{abstract}
Pulmonary artery injury (PAI) is rare, lethal clinical entity. Traumatic PAI is anatomically classified into transection/ rupture/laceration, pseudoaneurysm, dissection and fistula. In addition, traumatic PAI is clinically classified into two major categories: iatrogenic and non-iatrogenic, depending on the mechanism of the trauma. The frequency, clinical symptoms and treatment differ between the two clinical categories. If PAI can be managed appropriately and promptly in patients without cardiac arrest, the patient may be saved, as PAI can be easily controlled with appropriate procedures due to the low pressure in the PA circulation.
\end{abstract}

Keywords: Pulmonary artery, trauma, iatrogenic

\section{INTRODUCTION}

Pulmonary artery injury (PAI) is a rare, lethal clinical entity. Most vital emergencies involve proximal PAI. However, if PAI can be managed appropriately and promptly in patients without cardiac arrest, the patient may be saved, as PAI can be easily controlled with appropriate procedures due to the low pressure in the PA circulation, provided the injury site is small ${ }^{[1]}$. In this review article, traumatic PA is anatomically classified into four categories and clinically classified into two major categories: iatrogenic and non-iatrogenic, depending on the mechanism of the trauma. The frequency, clinical symptoms and treatment differ between the two clinical categories. The references are limited to reports in the English literature published since 1990 .

\footnotetext{
(a) (1)

(C) The Author(s) 2018. Open Access This article is licensed under a Creative Commons Attribution 4.0 International License (https://creativecommons.org/licenses/by/4.0/), which permits unrestricted use, sharing, adaptation, distribution and reproduction in any medium or format, for any purpose, even commercially, as long as you give appropriate credit to the original author(s) and the source, provide a link to the Creative Commons license, and indicate if changes were made.
}

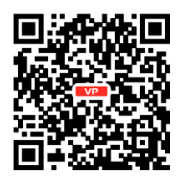




\section{ANATOMICAL CLASSIFICATION}

Anatomically, traumatic PAI is classified into transection/rupture/laceration, pseudoaneurysm, dissection and fistula.

A transection, rupture, disruption, perforation, tear or laceration of the PA is thought to be a near-complete tear through all layers of the PA due to trauma; however, there is no consistent definitive terminology ${ }^{[1]}$. Clinical symptoms due to such trauma include cardiac arrest or hemodynamic insufficiency due to massive hemorrhaging or cardiac tamponade, and dyspnea due to hemothorax or hemoptysis ${ }^{[1,2]}$. Chest pain due to concomitant thoracic cage injury has also been reported. Rarely, this PAI, which involves hemostasis by clotting, is incidentally found on enhanced computed tomography (CT) without specific symptoms, as whole-body enhanced CT is routinely performed in patients following a high-energy accident ${ }^{[3,4]}$.

A pseudoaneurysm is an encapsulated hematoma in communication with the lumen of a ruptured vessel. This may form when re-epithelialization of the perforation does not occur, and a delayed diagnosis can occur even 60 years later ${ }^{[5]}$. The pseudoaneurysm may stabilize and spontaneously resolve or expand and rupture, depending on the etiology, size and intravascular pressure ${ }^{[1]}$. A pseudoaneurysm can be asymptomatic or characterized by symptoms of hemoptysis, shortness of breath and chest pain ${ }^{[6,7]}$. An iatrogenic pseudoaneurysm of the PA is most common, followed by trauma-induced events. A pseudoaneurysm of the PA can also be congenital or have a non-traumatic cause, which includes infections and neoplasms $s^{[6,7]}$.

An arterial fistula is an abnormal connection between the artery and other lumen organs. If an abnormal connection between an artery and a vein occur, this is called as an arteriovenous (AV) fistula. In a trauma setting, arterial fistulas can be asymptomatic or characterized by right ventricular dysfunction, acute respiratory failure or transient ischemic attack (TIA ${ }^{[8-12]}$. Traumatically, fistulas occur between the PA and left atrium, internal mammary artery, aorta or pulmonary vein. Non-traumatic pulmonary AV fistulas can also be associated with hereditary hemorrhagic telangiectasia ${ }^{[13]}$. The initial clinical manifestations include thrombotic or embolic stroke, brain abscess and TIA but can also be asymptomatic in non-traumatic cases ${ }^{[13]}$. The clinical trial of cyanosis, exertional dyspnea and digital clubbing is common, but there have been no reports describing triads due to trauma ${ }^{[13]}$.

PA dissections (PADs) are created by the occurrence of a small tear in the tunica intima, which allows blood to enter and cause the intima layer to strip away from the media layer, in effect dividing the muscle layers of the vascular wall. The mechanism of blunt traumatic PAD is likely similar to that seen in the aorta as a result of shearing forces and differential deceleration of the mediastinum and the spine. However, unlike aortic dissection, PAD progresses rapidly and typically ruptures rather than developing a reentry site, which causes cardiogenic shock or sudden death, especially in non-traumatic cases with pulmonary hypertension ${ }^{[14]}$. Five major etiological groups can be identified: congenital malformation, infection or inflammation, acquired cardiac diseases, iatrogenic causes and trauma ${ }^{[15-17]}$. Traumatic PADs usually resolve or remain stable unless associated with pulmonary hypertension, in which case the risk of bleeding can be quite high ${ }^{[1,16]}$.

\section{IATROGENIC PAI}

The most common cause of PA ruptures and pseudoaneurysms is iatrogenic, with PA catheters being a particularly common culprit ${ }^{[2,18-20]}$. Other iatrogenic causes include intraoperative surgical procedures ${ }^{[21-24]}$, indwelling chest tubes ${ }^{[25,26]}$, pacemaker implantation ${ }^{[27]}$, central venous catheterization ${ }^{[28]}$ and Kirschner wire migration $^{[29]}$.

The incidence of PAI induced by catheters is not very high, averaging $0.01 \%-0.47 \%^{[2]}$. The mortality rate of PAI induced by catheter averages $50 \%$ but can be as high as $75 \%$ in anticoagulated patients. If death occurs, 
it is usually secondary to asphyxia rather than hypovolemia ${ }^{[2]}$. The initial presentation may be as obvious as massive pulmonary hemorrhaging or as subtle as a cough associated with minimal hemoptysis, or it may even be totally asymptomatic ${ }^{[30]}$.

When catheter-induced PAI happens during insertion of a fluoroscope, it is relatively easy to retract the PA catheter a few centimeters and re-inflate the balloon under direct vision. It may therefore be possible to stop the bleeding ${ }^{[2]}$. Additional diagnostic angiography and embolization also can be easily performed at that point.

In addition to treatments for PAI, the patient may need selective intubation to obtain lung isolation in accordance with clinical symptoms. Lung isolation can be performed with different techniques, including selective intubation with a standard endotracheal tube, bronchial blocker or double-lumen tube (DLT) ${ }^{[2]}$. A bronchial blocker can be used for lung separation when a DLT is not immediately available or when it is difficult to insert the DLT. Bronchial blockers can be used to tamponade the bleeding side while waiting for diagnostic and therapeutic interventions. The most important aspects of treatment are lung isolation using selective intubation, bronchial blockers, or DLT as a temporary measure; rapid movement is important for more definitive therapy as it can avoid clotting of the entire lung on one side, which effectively causes pneumonectomy. Surgery, including pulmonary artery ligature, segmentectomy, lobectomy or pneumonectomy, is reserved for extreme cases, since these procedures are technically challenging and entail high morbidity ${ }^{[2]}$.

\section{NON-IATROGENIC PAI}

A majority of non-iatrogenic PAI cases occur due to chest trauma; however, most chest trauma cases do not involve PAI. PAI accounts for a small percentage of thoracic trauma cases. Epidemiologically, Kulshrestha et al.$^{[31]}$ reported 102 patients sustaining cardiac injuries over a 4-year period. There were 45 blunt trauma, 36 stab injuries, and 21 gunshot injuries ${ }^{[31]}$. The injury involved the ventricle in 85 patients, atrium in 7 and the PA in 5 (5\%) and resulted in crush injury to the heart in the remaining 5 cases. Thirty-three patients $(32.3 \%)$ died at the scene, and 58 (56.9\%) died during transportation. Only 11 patients (10.8\%) reached the hospital alive, and 10 of these survived following thoracotomy and repair of the cardiac injury. The patients with ventricular injuries had a greater prehospital mortality than those with atrial or PA injuries.

Deneuville ${ }^{[32]}$ reported 88 cases of penetrating chest trauma, focusing on non-iatrogenic PAIs. Of these 88 cases, 6 with PAI reached the hospital alive ${ }^{[32]}$. All cases underwent urgent operation, and 4 survived. The mortality appears to be high in patients presenting with complex lesions involving vascular and pulmonary structures. As a result, they concluded that isolated injuries of the PA were amenable to surgical repair and had a good prognosis if the patients arrived at the hospital alive.

We summarized the cases of non-iatrogenic PAI in Tables 1 and 2. Most cases were reported as case reports, except for the findings of Deneuville ${ }^{[32]}$. Penetrating injuries were more frequent than blunt ones. Similar to Deneuville ${ }^{[32]}, 46 / 50$ (92\%) cases survived. The diagnosis was made based on intraoperative findings, enhanced CT or pulmonary arteriography. The main treatment method was surgery or an interventional approach. These findings suggest that if hemorrhaging is not noted and the vital signs are stable, conservative treatment can be selected. There are no strict guidelines concerning the management of PAI, and the preferred approach depends on the lesion, patient and institution ${ }^{[1]}$.

\section{CONCLUSION}

PAI is a rare, lethal clinical entity; most vital emergencies involve proximal PAI. Anatomically, traumatic PAI is classified into transection/rupture/laceration, pseudoaneurysm, dissection and fistula. Iatrogenic 
Table 1. Cases of non-iatrogenic injury of the pulmonary artery since 1990

\begin{tabular}{|c|c|c|c|c|c|c|c|c|c|c|c|c|}
\hline No. & Reporter & Year & $\begin{array}{c}\text { Age } \\
\text { (year) }\end{array}$ & Gender & $\begin{array}{l}\text { Type of } \\
\text { injury }\end{array}$ & $\begin{array}{l}\text { Cause of } \\
\text { injury }\end{array}$ & $\begin{array}{l}\text { Type of } \\
\text { injury }\end{array}$ & Symptom & Treatment & Outcome & Arrest & Other \\
\hline 1 & $\begin{array}{l}\text { Demondion } \\
\text { et } a l_{.}^{[33]}\end{array}$ & 2016 & 27 & Male & Blunt & $\begin{array}{l}\text { Snowmobile } \\
\text { accident }\end{array}$ & Rupture & $\begin{array}{l}\text { Mediastinal } \\
\text { hematoma }\end{array}$ & Conservative & Survive & None & \\
\hline 2 & $\begin{array}{l}\text { Maury } \\
\text { et al. } .^{[34]}\end{array}$ & 2015 & 51 & Male & Blunt & $\begin{array}{l}\text { Traffic } \\
\text { accident }\end{array}$ & Rupture & Hemothorax & Suture & Survive & None & \\
\hline 3 & Lin et al. ${ }^{[35]}$ & 2014 & 23 & Male & Blunt & $\begin{array}{l}\text { Traffic } \\
\text { accident }\end{array}$ & Rupture & Hemothorax & Ligation & Dead & None & MOF \\
\hline 4 & $\begin{array}{l}\text { Muthialu } \\
\text { et } a .^{[36]}\end{array}$ & 2013 & 5 & Female & Blunt & $\begin{array}{l}\text { Traffic } \\
\text { accident }\end{array}$ & Rupture & Hemothorax & $\begin{array}{l}\text { Suture \& } \\
\text { lobectomy }\end{array}$ & Survive & None & \\
\hline 5 & $\begin{array}{l}\text { Vendrell and } \\
\text { Gahide }^{[3]}\end{array}$ & 2010 & 42 & Female & Blunt & $?$ & Rupture & Hemothorax & Conservative & Survive & None & \\
\hline 6 & $\begin{array}{l}\text { Pereira and } \\
\text { Narrod }^{[37]}\end{array}$ & 2009 & 55 & Female & Blunt & $\begin{array}{l}\text { Traffic } \\
\text { accident }\end{array}$ & Rupture & Hemothorax & Suture & Survive & None & \\
\hline 7 & $\begin{array}{l}\text { Kanani } \\
\text { et al. }{ }^{[38]}\end{array}$ & 2002 & 31 & Male & Blunt & $\begin{array}{l}\text { Traffic } \\
\text { accident }\end{array}$ & Rupture & Hemothorax & Suture & Survive & None & \\
\hline 8 & $\begin{array}{l}\text { Ambrose } \\
\text { et al. }{ }^{[39]}\end{array}$ & 2000 & 69 & Male & Blunt & $\begin{array}{l}\text { Traffic } \\
\text { accident }\end{array}$ & Rupture & Hemothorax & Suture & Survive & None & \\
\hline 9 & $\begin{array}{l}\text { Weltman } \\
\text { et al. }{ }^{[40]}\end{array}$ & 1999 & 69 & Male & Blunt & $\begin{array}{l}\text { Traffic } \\
\text { accident }\end{array}$ & Rupture & Hemothorax & Suture & Survive & None & \\
\hline 10 & $\begin{array}{l}\text { Clements } \\
\text { et al. }{ }^{[41]}\end{array}$ & 1997 & 42 & Female & Blunt & $\begin{array}{l}\text { Traffic } \\
\text { accident }\end{array}$ & Rupture & Tamponade & Suture & Survive & None & \\
\hline 11 & $\begin{array}{l}\text { Daon and } \\
\text { Gorton }^{[42]}\end{array}$ & 1997 & 44 & Female & Blunt & $\begin{array}{l}\text { Traffic } \\
\text { accident }\end{array}$ & Rupture & Hemothorax & Suture & Survive & None & \\
\hline 12 & $\begin{array}{l}\text { Katz and } \\
\text { Groskin }^{[43]}\end{array}$ & 1993 & 27 & Female & Blunt & $\begin{array}{l}\text { Traffic } \\
\text { accident }\end{array}$ & Rupture & Hemothorax & Suture & Survive & None & \\
\hline 13 & $\begin{array}{l}\text { Ohsaka } \\
\text { et al. }{ }^{[44]}\end{array}$ & 2015 & 91 & Male & Penetrating & Sord & Rupture & Hemothorax & Suture & Survive & PEA & \\
\hline 14 & $\begin{array}{l}\text { Greberski } \\
\text { et al. }{ }^{[45]}\end{array}$ & 2015 & 36 & Male & Penetrating & Knife & Rupture & Hemothorax & Suture & Survive & None & \\
\hline 15 & $\begin{array}{l}\text { Senanayake } \\
\text { et al. }{ }^{[46]}\end{array}$ & 2012 & 54 & Male & Penetrating & Stab & Rupture & Hemothorax & Suture & Survive & PEA & \\
\hline 16 & $\begin{array}{l}\text { Sanchez } \\
\text { et al. }{ }^{[47]}\end{array}$ & 2010 & 31 & Male & Penetrating & Stab & Rupture & Hemothorax & Suture & Survive & None & \\
\hline 17 & $\begin{array}{l}\text { Atalay } \\
\text { et al. }{ }^{[48]}\end{array}$ & 2010 & 18 & Male & Penetrating & Gun & Rupture & Hemothorax & Suture & Survive & None & \\
\hline 18 & Deneuville $^{[32]}$ & 2000 & 32 & Male & Penetrating & Knife & Rupture & Hemothorax & Suture & Survive & None & \\
\hline 19 & Deneuville ${ }^{[32]}$ & 2000 & 37 & Male & Penetrating & Shotgun & Rupture & Hemothorax & Suture & Survive & None & \\
\hline 20 & Deneuville $^{[32]}$ & 2000 & 24 & Male & Penetrating & Knife & Rupture & Hemothorax & Suture & Survive & None & \\
\hline 21 & Deneuville $^{[32]}$ & 2000 & 22 & Male & Penetrating & Shotgun & Rupture & Hemothorax & Pneumonectomy & Dead & Yes & \\
\hline 22 & Deneuville $^{[32]}$ & 2000 & 55 & Male & Penetrating & Bull horn & Rupture & Hemothorax & Pneumonectomy & Dead & Yes & \\
\hline 23 & Deneuville $^{[32]}$ & 2000 & 44 & Male & Penetrating & Knife & Rupture & Hemothorax & Suture & Survive & None & \\
\hline 24 & $\begin{array}{l}\text { Babatasi } \\
\text { et al. }{ }^{[49]}\end{array}$ & 1999 & 69 & Male & Penetrating & Gun & Rupture & $\begin{array}{l}\text { Mediastinal } \\
\text { hematoma }\end{array}$ & Suture & Survive & None & \\
\hline 25 & Kiss et $a .^{[50]}$ & 1999 & 34 & Woman & Penetrating & Gun & Rupture & Tamponade & Suture & Survive & None & \\
\hline 26 & $\operatorname{Jain}^{[51]}$ & 1998 & 7 & Male & Penetrating & Air gun & Rupture & Tamponade & Suture & Survive & PEA & \\
\hline 27 & Goel et al. ${ }^{[52]}$ & 2013 & 58 & Female & Blunt & $\begin{array}{l}\text { Traffic } \\
\text { accident }\end{array}$ & Pseudoaneurysm & No specific & Conservative & Survive & None & \\
\hline 28 & $\begin{array}{l}\text { Sridhar } \\
\text { et al. } .^{[53]}\end{array}$ & 2010 & 32 & Male & Blunt & $?$ & Pseudoaneurysm & No specific & Embolization & Survive & None & \\
\hline 29 & $\begin{array}{l}\text { Reade } \\
\text { et al. }{ }^{[54]}\end{array}$ & 2006 & 57 & Male & Blunt & $\begin{array}{l}\text { Traffic } \\
\text { accident }\end{array}$ & Pseudoaneurysm & No specific & Conservative & Survive & None & \\
\hline 30 & $\begin{array}{l}\text { Kasai and } \\
\text { Kobayashi }^{[55]}\end{array}$ & 1992 & 17 & Male & Blunt & $\begin{array}{l}\text { Traffic } \\
\text { accident }\end{array}$ & Pseudoaneurysm & No specific & Lobectomy & Survive & None & \\
\hline 31 & Goel et al. ${ }^{[52]}$ & 2013 & 32 & Male & Penetrating & Gun & Pseudoaneurysm & No specific & Conservative & Survive & None & \\
\hline 32 & $\begin{array}{l}\text { Quartey and } \\
\text { Jessie }^{[56]}\end{array}$ & 2011 & 21 & Male & Penetrating & Gun & Pseudoaneurysm & No specific & Coil embolization & Survive & None & \\
\hline 33 & Blanié et $a l .{ }^{[57]}$ & 2011 & 39 & Male & Penetrating & $\begin{array}{l}\text { Circular } \\
\text { saw }\end{array}$ & Pseudoaneurysm & No specific & Pericardial patch & Survive & None & \\
\hline 34 & Rai et al. ${ }^{[58]}$ & 2010 & 28 & Woman & Penetrating & Gun & Pseudoaneurysm & No specific & Coil embolization & Survive & Yes & \\
\hline 35 & $\begin{array}{l}\text { Maddali } \\
\text { et al. } .^{[59]}\end{array}$ & 2007 & 35 & Male & Penetrating & Knife & Pseudoaneurysm & No specific & Suture & Survive & None & \\
\hline 36 & Khan et al. ${ }^{[60]}$ & 2005 & 50 & Male & Penetrating & Gun & Pseudoaneurysm & No specific & Coil embolization & Survive & None & \\
\hline 37 & $\begin{array}{l}\text { Dimarakis } \\
\text { et al. }{ }^{[61]}\end{array}$ & 2005 & 29 & Male & Penetrating & Knife & Pseudoaneurysm & No specific & Coil embolization & Survive & None & \\
\hline 38 & Block et al. ${ }^{[7]}$ & 2004 & 40 & Male & Penetrating & Gun & Pseudoaneurysm & No specific & Coil embolization & Survive & None & \\
\hline
\end{tabular}




\begin{tabular}{|c|c|c|c|c|c|c|c|c|c|c|c|c|}
\hline & $\begin{array}{l}\text { de Jonge } \\
\text { et al. }{ }^{[62]}\end{array}$ & 2003 & 57 & Male & Penetrating & Knife & Pseudoaneurysm & No specific & Coil embolization & Survive & None & \\
\hline 40 & $\begin{array}{l}\text { Donaldson } \\
\text { and Ngo- } \\
\text { Nonga }{ }^{[63]}\end{array}$ & 2002 & 17 & Male & Penetrating & Gun & Pseudoaneurysm & No specific & Lobectomy & Survive & Yes & CPC4 \\
\hline 41 & $\begin{array}{l}\text { Savage } \\
\text { et al. }{ }^{[64]}\end{array}$ & 1999 & 49 & Male & Penetrating & Gun & Pseudoaneurysm & No specific & Coil embolization & Survive & None & \\
\hline 42 & $\begin{array}{l}\text { Hubler } \\
\text { et al. }{ }^{[65]}\end{array}$ & 1997 & 20 & Male & Penetrating & Knife & Pseudoaneurysm & No specific & Lobectomy & Survive & None & \\
\hline 43 & 3 Huet et al. ${ }^{[66]}$ & 1996 & 29 & Male & Penetrating & Gun & Pseudoaneurysm & No specific & Stent & Survive & None & \\
\hline & $\begin{array}{l}4 \text { Giglioli } \\
\text { et al. }{ }^{[10]}\end{array}$ & 2013 & 46 & Female & Blunt & $?$ & $\begin{array}{l}\text { Fistula } \\
\text { (aortopulmonary) }\end{array}$ & $\begin{array}{l}\text { Right cardiac } \\
\text { failure }\end{array}$ & Pericardial patch & Survive & None & \\
\hline 45 & $\begin{array}{l}\text { Rrapo } \\
\text { et al } !^{[11]}\end{array}$ & 2013 & 20 & Male & Penetrating & Gun & $\begin{array}{l}\text { Fistula } \\
\text { (pulmonary) }\end{array}$ & ARDS & Pericardial patch & Survive & None & \\
\hline 46 & $\begin{array}{l}\text { Roshanali } \\
\text { et al. }{ }^{[12]}\end{array}$ & 2012 & 48 & Female & Penetrating & $\begin{array}{l}\text { Missile } \\
\text { debris }\end{array}$ & $\begin{array}{l}\text { Fistula } \\
\text { (pulmonary) }\end{array}$ & TIA & Plug occlusion & Survive & None & \\
\hline 47 & $\begin{array}{l}\text { Howell } \\
\text { et } a l^{[67]}\end{array}$ & 2004 & 24 & Male & Penetrating & Knife & $\begin{array}{l}\text { Fistula } \\
\text { (aortopulmonary) }\end{array}$ & No specific & Operation & Survive & None & \\
\hline 48 & $\begin{array}{l}\text { Kerr and } \\
\text { Sauter }\end{array}$ & 1993 & 35 & Male & Penetrating & Knife & $\begin{array}{l}\text { Fistula } \\
\text { (pulmonary) }\end{array}$ & $\begin{array}{l}\text { Short of } \\
\text { breath }\end{array}$ & Embolization & Survive & None & \\
\hline & $\begin{array}{l}\text { Almdahl } \\
\text { et al. }{ }^{[16]}\end{array}$ & 2014 & 46 & Female & Blunt & ? & Dissection & No specific & Conservative & Survive & None & \\
\hline & $\begin{array}{l}\text { Chung } \\
\text { et } a l^{[17]}\end{array}$ & 2009 & 53 & Male & Blunt & $\begin{array}{l}\text { Boat } \\
\text { accident }\end{array}$ & Dissection & No specific & Nitric oxide & Dead & None & \\
\hline
\end{tabular}

?: not described; ARDS: acute respiratory distress syndrome; TIA: transient ischemic attack; MOF: multiple organ failure; PEA: pulseless electrical activity; CPC: cerebral performance category; PAI: pulmonary artery injury

Table 2. Summary of non-iatrogenic injury of the pulmonary artery since 1990

\begin{tabular}{lll}
\hline Total & & \multicolumn{1}{c}{$\mathbf{5 0}$ cases } \\
\hline Age, years & Range & $5-91$ \\
Gender & Average & 38.4 \\
& Male & $40(80 \%)$ \\
Type of injury & Female & $10(20 \%)$ \\
& Blunt & $19(38 \%)$ \\
Cause of injury & Penetrating & $31(62 \%)$ \\
& Gun & $15(30 \%)$ : shot gun, air gun include \\
& Traffic accident & $13(32 \%)$ \\
& Knife & $10(20 \%)$ \\
Type of PAI & Others & $9(18 \%)$ \\
& Rupture & $26(52 \%)$ \\
& Pseudoaneurysm & $17(34 \%)$ \\
& Fistula & $5(10 \%)$ \\
Symptom & Dissection & $2(4 \%)$ \\
& Hemothorax & $21(42 \%)$ \\
& No specific & $20(40 \%)$ \\
& Tamponade & $3(6 \%)$ \\
Treatment & Others & $3(6 \%)$ \\
& Surgical sutures & $22(44 \%)$ \\
& Endovascular & $10(20 \%):$ include coil, stent and other materials \\
& Conservative & $6(12 \%)$ \\
& Other surgical maneuver & $10(20 \%)$ \\
Survival & Other treatment & $2(4 \%)$ \\
\hline & Number and rate & $46(92 \%)$ \\
\hline
\end{tabular}

PAl: pulmonary artery injury

procedures are the most common cause of iatrogenic PAI rupture and pseudoaneurysm, with PA catheters being a particularly common culprit. Non-iatrogenic PAIs occur due to chest trauma but most chest trauma does not involve PAI. Penetrating injuries were more frequent than blunt injuries. The diagnosis was made based on intraoperative findings, enhanced CT or pulmonary arteriography. The main treatment method was surgery or an interventional approach. If PAI can be managed appropriately and promptly in patients without cardiac arrest, the patient may be saved. 


\section{DECLARATIONS}

\section{Authors' contributions}

Designed the study, gathered data and wrote the manuscript: Yanagawa $Y$

Gave technical support, conceptual advice and edited the manuscript: Ishikawa K, Nagasawa H, Takeuchi I, Jitsuiki K, Ohsaka H, Omori K

\section{Financial support and sponsorship}

This manuscript obtains financial support from the Ministry of Education, Culture, Sports, Science and Technology - Supported Program for the Strategic Research Foundation at Private Universities, 2015-2019 concerning (The constitution of total researching system for comprehensive disaster, medical management, corresponding to wide-scale disaster).

\section{Conflicts of interest}

The authors declare no conflicts of interest in association with this study.

\section{Patient consent}

Not applicable.

\section{Ethics approval}

This review article was approved by the review board of Juntendo Shizuoka Hospital, and all examinations were conducted according to the standards of good clinical practice and the Helsinki Declaration.

\section{Copyright}

(c) The Author(s) 2018.

\section{REFERENCES}

1. Abbas AE. Traumatic injury of the pulmonary artery: transection, rupture, pseudoaneurysm, or dissection? Sometimes semantics do matter. J Thorac Cardiovasc Surg 2016;152:1437-8.

2. Bussières JS. Iatrogenic pulmonary artery rupture. Curr Opin Anaesthesiol 2007;20:48-52.

3. Vendrell JF, Gahide G. Right pulmonary artery transection following blunt chest trauma. Eur J Cardiothorac Surg 2010;38:802.

4. Wurmb TE, Bernhard M. Total-body CT for initial diagnosis of severe trauma. Lancet 2016;388:636-8.

5. Surov A, Spielmann RP, Werdan K, Buerke M, Behrmann C. A late presentation of giant traumatic pulmonary artery aneurysm. Circulation 2010;122:2581-2.

6. Chen Y, Gilman MD, Humphrey KL, Salazar GM, Sharma A, Muniappan A, Shepard JO, Wu CC. Pulmonary artery pseudoaneurysms: clinical features and CT findings. AJR Am J Roentgenol 2017;208:84-91.

7. Block M, Lefkowitz T, Ravenel J, Leon S, Hannegan C. Endovascular coil embolization for acute management of traumatic pulmonary artery pseudoaneurysm. J Thorac Cardiovasc Surg 2004;128:784-5.

8. Abou Zahr R, Hellenbrand WE, Asnes JD. Iatrogenic left pulmonary artery to left atrium fistula. Catheter Cardiovasc Interv 2015;85:847-9.

9. Yan Y, Tweddale BA, Trerotola SO. Internal mammary artery-to-pulmonary artery and vein fistula acquired after video-assisted thoracoscopic surgery and pleurodesis. J Vasc Interv Radiol 2013;24:1759-61.

10. Giglioli C, Cecchi E, Angelotti P, Venditti F, Calabretta R, Scheggi V, Alterini B, Stefano P. Aortopulmonary fistula presenting with right ventricular dysfunction following blunt chest trauma. J Card Surg 2013;28:713.

11. Rrapo E, Lube MW, Smith CP. Right axillary artery bullet embolus and the formation of a pulmonary arteriovenous fistula after a gunshot wound to the back. Am Surg 2013;79:E172-4.

12. Roshanali F, Mandegar MH, Oraii S. Traumatic pulmonary arteriovenous fistula may be misdiagnosed with residual shunt after patent foramen ovale closure. BMJ Case Rep 2012;2012:bcr2012006802.

13. Yamakuchi M, Tanaka S, Tomosugi T, Moroki K, Yamada M, Toujou H, Uetsuhara K, Maruyama I. Pulmonary arteriovenous fistula manifesting as amaurosisfugax--case report. Neurol Med Chir (Tokyo) 2000;40:264-7.

14. Zhang C, Huang X, Li S, Yao H, Zhang B. Pulmonary artery dissection: a fatal complication of pulmonary hypertension. Case Rep Med 2016;2016:4739803.

15. Adodo DK, Kloeckner M, Bergoend E, Couëtil JP. Pulmonary artery dissection: a case treated by homograft replacement. Ann Thorac Surg 2017;103:e47-9.

16. Almdahl SM, Jakobsen $\varnothing$, Skattør TH. Dissection of the right pulmonary artery after blunt trauma. Eur $J$ Cardiothorac Surg 
2014;46:141-2.

17. Chung JH, Mullins CD, Manchanda V, Gunn ML, Stern EJ. Pulmonary artery intimal injury associated with blunt trauma. Emerg Radiol 2009;16:497-9.

18. Atreya AR, Arora S, Valania G. Pulmonary artery rupture with pseudoaneurysm formation secondary to Swan-Ganz catheter balloon inflation. Acute Card Care 2015;17:77-9.

19. Inami T, Kataoka M, Shimura N, Ishiguro H, Yanagisawa R, Kawakami T, Fukuda K, Yoshino H, Satoh T. Incidence, avoidance, and management of pulmonary artery injuries in percutaneous transluminal pulmonary angioplasty. Int J Cardiol 2015;201:35-7.

20. Ejiri K, Ogawa A, Matsubara H. Bail-out technique for pulmonary artery rupture with a covered stent in balloon pulmonary angioplasty for chronic thromboembolic pulmonary hypertension. JACC Cardiovasc Interv 2015;8:752-3.

21. Berry MF. Pulmonary artery bleeding during video-assisted thoracoscopic surgery: intraoperative bleeding and control. Thorac Surg Clin 2015;25:239-47.

22. Mei J, Pu Q, Liao H, Ma L, Zhu Y, Liu L. A novel method for troubleshooting vascular injury during anatomic thoracoscopic pulmonary resection without conversion to thoracotomy. Surg Endosc 2013;27:530-7.

23. Cardillo G, Carleo F, DI Martino M, Ciamberlano B, Ialongo P, Cusumano G, Denitza Tinti M, Ricci A, Cafarotti S. Control of major pulmonary artery bleeds with a gelatin matrix-thrombin solution: a retrospective analysis. J Cardiovasc Surg (Torino) 2017;58:904-8.

24. Péterffy A, Henze A. Haemorrhagic complications during pulmonary resection. A retrospective review of 1428 resections with 113 haemorrhagic episodes. Scand J Thorac Cardiovasc Surg 1983;17:283-7.

25. Bozzani A, Arici V, Bellinzona G, Pirrelli S, Forni E, Odero A. Iatrogenic pulmonary artery rupture due to chest-tube insertion. Tex Heart Inst J 2010;37:732-3.

26. Sundaramurthy SR, Moshinsky RA, Smith JA. Non-operative management of tube thoracostomy induced pulmonary artery injury. Interact Cardiovasc Thorac Surg 2009;9:759-60.

27. Tokue H, Tokue A, Morita H, Tsushima Y. Successful interventional management for pulmonary arterial injury secondary to pacemaker implantation. Case Rep Cardiol 2016;2016:4340193.

28. Hunt LB, Olshansky B, Hiratzka LF. Cardiac tamponade caused by pulmonary artery perforation after central venous catheterization. JPEN J Parenter Enteral Nutr 1984;8:711-3.

29. Liu HP, Chang CH, Lin PJ, Chu JJ, Hsieh HC, Chang JP, Hsieh MC. Pulmonary artery perforation after Kirschner wire migration: case report and review of the literature. J Trauma 1993;34:154-6.

30. Poplausky MR, Rozenblit G, Rundback JH, Crea G, Maddineni S, Leonardo R. Swan-Ganz catheter-induced pulmonary artery pseudoaneurysm formation: three case reports and a review of the literature. Chest 2001;120:2105-11.

31. Kulshrestha P, Das B, Iyer KS, Sampath KA, Sharma ML, Rao IM, Venugopal P. Cardiac injuries--a clinical and autopsy profile. $J$ Trauma 1990;30:203-7.

32. Deneuville M. Injury of the pulmonary artery and its branches due to penetrating chest trauma. Ann Vasc Surg 2000;14:463-7.

33. Demondion P, Bellemare P, El-Hamamsy I. Conservative management of an intrapericardial contained rupture of the right pulmonary artery in blunt trauma: a good idea? J Thorac Cardiovasc Surg 2016;152:1435-6.

34. Maury JM, Deslandes L, Oheix S, David JS. Acute traumatic right pulmonary artery rupture in blunt trauma. Intensive Care Med 2015;41:134-5.

35. Lin YY, Tiu CM, Chen JD, Chou YH, Hsueh HC, Tseng TK, Lee MH, Chang CY. Segmental pulmonary artery transection after blunt trauma. J Chin Med Assoc 2014;77:389-92.

36. Muthialu N, Hoskote A, Deshpande R, Lister P. Right pulmonary hilar pedicle injury secondary to blunt chest trauma in a child. Asian Cardiovasc Thorac Ann 2013;21:235-8.

37. Pereira SJ, Narrod JA. Repair of right pulmonary artery transection after blunt trauma. Ann Thorac Surg 2009;87:939-40.

38. Kanani N, Ting P, Weber B, Gray RR, Maitland A. Blunt trauma resulting in systemic arterial and pulmonary artery injury: case report. Can Assoc Radiol J 2002;53:141-3.

39. Ambrose G, Barrett LO, Angus GL, Absi T, Shaftan GW. Main pulmonary artery laceration after blunt trauma: accurate preoperative diagnosis. Ann Thorac Surg 2000;70:955-7.

40. Weltman DI, Baykal A, Zhang D. CT diagnosis of laceration of the main pulmonary artery after blunt trauma. AJR Am J Roentgenol 1999;173:1361-2.

41. Clements RH, Wagmeister LS, Carraway RP. Blunt intrapericardial rupture of the pulmonary artery in a surviving patient. Ann Thorac Surg 1997;64:258-60.

42. Daon E, Gorton ME. Traumatic disruption of the innominate and right pulmonary arteries: case report. J Trauma 1997;43:701-2.

43. Katz DS, Groskin SA. Pulmonary artery laceration and tension pneumothorax in blunt chest trauma. J Thorac Imaging 1993;8:156-8.

44. Ohsaka H, Yanagawa Y, Miyasaka Y, Okamoto K. Successful treatment of a penetrating pulmonary artery injury caused by a Japanese sword in a patient transported by a physician-staffed helicopter. J Emerg Trauma Shock 2015;8:125-6.

45. Greberski K, Bugajski P, Rzymski S, Jarząbek R, Olczak B, Kalawski R. Penetrating thoracic injuries - treatment of two patients after suicide attempts. Kardiochir Torakochirurgia Pol 2015;12:62-4.

46. Senanayake EL, Jeyatheesan J, Rogers V, Wilson IC, Graham TR. Stab to the chest causing severe great vessel injury. Ann Thorac Surg 2012;94:1716-8

47. Sanchez GP, Peng EW, Marks R, Sarkar PK. 'Scoop and run' strategy for a resuscitative sternotomy following unstable penetrating chest injury. Interact Cardiovasc Thorac Surg 2010;10:467-8.

48. Atalay HH, Demirtürk OS, Kiliç D, Türköz R. Gunshot wound of the main pulmonary artery: a case report. J Thorac Cardiovasc Surg 
2010;139:e17-8.

49. Babatasi G, Massetti M, Bhoyroo S, Le Page O, Khayat A. Pulmonary artery bullet injury following thoracic gunshot wound. Eur $J$ Cardiothorac Surg 1999;15:87-90.

50. Kiss SS, Tóth P, Kollár S, Nábrádi Z, Bóni J. Five-year study on the injury of the great thoracic vessels after penetrating chest injury. Acta Chir Hung 1999;38:75-8.

51. Jain AK. Survival following cardiac tamponade and arrest in a paediatric patient with penetrating trauma to pulmonary artery. Paediatr Anaesth 1998;8:345-8.

52. Goel S, Kumar A, Gamanagatti S, Gupta A. Spontaneous resolution of post-traumatic pulmonary artery pseudoaneurysm: report of two cases. Lung India 2013;30:203-5.

53. Sridhar SK, Sadler D, McFadden SD, Ball CG, Kirkpatrick AW. Percutaneous embolization of an angiographically inaccessible pulmonary artery pseudoaneurysm after blunt chest trauma: a case report and review of the literature. J Trauma 2010;69:729.

54. Reade CC, Jenkins NL, Bard MR, Kuszyk BS, Koutlas TC, Rotondo MF. Immediate diagnosis and nonoperative treatment of a pulmonary artery pseudoaneurysm after blunt traumatic injury. J Trauma 2006;60:894-6.

55. Kasai T, Kobayashi K. Bilateral pseudoaneurysms of the pulmonary arteries caused by blunt chest injury. Intensive Care Med 1992;18:51-2.

56. Quartey B, Jessie E. Pulmonary artery and vein pseudoaneurysm after gunshot wound to the chest. J Emerg Trauma Shock 2011;4:313-6.

57. Blanié A, Fadel E, Duranteau J. Left pulmonary artery transection after penetrating thoracic trauma. $J$ Trauma 2011;71:1479.

58. Rai VK, Malireddy K, Dearmond D, Myers J, Dent DL. Traumatic pseudoaneurysm of the pulmonary artery. J Trauma 2010;69:730.

59. Maddali MM, Zacharias S, Adhikari RK, Rajakumar MC, Ahmed AR. Traumatic pseudoaneurysm of pulmonary artery. Asian Cardiovasc Thorac Ann 2007;15:362-3.

60. Khan AA, Bauer TL, Garcia MJ, Panasuk DB, Davies AL. Angiographic embolization of a traumatic pulmonary pseudoaneurysm. Ann Thorac Surg 2005;79:2136-8.

61. Dimarakis I, Thorpe JA, Papagiannopoulos K. Successful treatment of a posttraumatic pulmonary artery pseudoaneurysm with coil embolization. Ann Thorac Surg 2005;79:2134-6.

62. de Jonge I, Vahl A, van der Hulst V. Coil embolization of a left pulmonary artery pseudoaneurysm after penetrating injury. $J$ Endovasc Ther 2003;10:681-3.

63. Donaldson B, Ngo-Nonga B. Traumatic pseudoaneurysm of the pulmonary artery: case report and review of the literature. Am Surg 2002;68:414-6.

64. Savage C, Zwischenberger JB, Ventura KC, Wittich GR. Hemoptysis secondary to pulmonary pseudoaneurysm 30 years after a gunshot wound. Ann Thorac Surg 2001;71:1021-3.

65. Hubler B, Earls JP, Stevens K. Traumatic pulmonary arterial and venous pseudoaneurysms. AJR Am J Roentgenol 1997;169:1354.

66. Huet N, Rodiere M, Badet M, Michoud M, Brichon PY, Ferretti G, Thony F. Covered stent and coils embolization of a pulmonary artery pseudoaneurysm after gunshot wound. Cardiovasc Intervent Radiol 2016;39:778-81.

67. Howell A, Brown R, Ashley DW, Williams J 4th, Lane JE. Aortopulmonary fistula from penetrating thoracic trauma. $J$ Trauma 2004;57:1374.

68. Kerr A, Sauter D. Acquired traumatic pulmonary arteriovenous fistula: case report. J Trauma 1993;35:484-6. 